Gutiérrez-Lestón, C., Pérez-Escoda, N., Reguant, M. y Eroles, M. (2020). Innovación de educación emocional en el ocio educativo: el Método La Granja. Revista de Investigación Educativa, 38(2), 495-513.

DOI: http://dx.doi.org/10.6018/rie.405721

\title{
Innovación de educación emocional en el ocio educativo: el Método La Granja
}

\author{
Emotional education. La Granja Method: an innovation in \\ educational leisure
}

\author{
Cristina Gutiérrez-Lestón*, Nuria Pérez-Escoda **, Mercedes Reguant** y Marta Eroles* \\ * La Granja Ability Training Center (España). \\ ** Departamento MIDE. Facultat d'Educación. Universitat de Barcelona (España)
}

\begin{abstract}
Resumen
En este trabajo se presenta la aportación en educación emocional del Método La Granja, aplicado en el casal de verano realizado en la granja escuela de Santa María de Palautordera el verano de 2017. Se trata de una experiencia de educación no formal propuesta para favorecer el aprendizaje desde la emoción y la experimentación. El objetivo principal es valorar el efecto de la participación de 127 niños y niñas entre 8 y 14 años en términos de mejora de sus competencias emocionales, así como su percepción sobre la utilidad de lo aprendido. Sigue un paradigma pragmático que combina datos recogidos por distintas vías de aproximación. Incluye la aplicación del Cuestionario de Desarrollo Emocional (CDE-9-13 y CDE-SEC, del GROP) antes y al final, más un cuestionario de preguntas abiertas como postest.

En la competencia emocional global, así como sus cinco dimensiones: conciencia, regulación, autonomía, competencias sociales y competencias de vida y bienestar, las puntuaciones aumentan, después de su participación en el Casal, esas diferencias resultaron significativas en todos los casos excepto en la última dimensión. Según los análisis lexicométrico y del contenido de las preguntas abiertas, hay coincidencias entre ambas observaciones, cuantitativas y el significado otorgado a la experiencia en términos de competencias emocionales. La discusión y conclusiones más relevantes giran en torno a la importancia de la reflexión como potenciador del aprendizaje, la duración e intensidad de las intervenciones y la alineación
\end{abstract}

Correspondencia: Núria Pérez-Escoda, nperezescoda@ub.edu, Pg. de la Vall d'Hebron, 171, 08035 Barcelona (España). 
de todos los recursos empleados que interactúan de forma sinérgica en torno al logro de los objetivos, temas recogidos ampliamente en la literatura científica.

Palabras clave: educación no formal; educación y ocio; innovación educacional; desarrollo emocional.

\begin{abstract}
This paper presents an innovative methodology in the emotional education field, La Granja, which was developed during a summer camp celebrated in 2017 in Santa María de Palautordera (Barcelona-Spain). It is a non-formal education experience proposed to promote learning from the emotions and experiences. The main goal is to assess the effect of this method on 127 children, between 8 and 14 years of age, in terms of improving their emotional competencies, as well as their perception of the usefulness of what they have learned. It follows a pragmatic paradigm that combines data collected by different approaches. It includes the application of the Emotional Development Questionnaire (CDE-9-13 and CDE-SEC, of the GROP) before and at the end of the summer camp, plus the ad hoc survey with open questions as post-test.

In the global emotional competence, as well as its five dimensions: awareness, emotional regulation, emotional autonomy, social competencies, and life and wellbeing competencies, the scores increase, after their participation in the summer camp. These differences were significant in all cases except in the last dimension. According to the lexicometric analysis and the content of the open questions, there are coincidences between both quantitative observations and the meaning given to the experience in terms of emotional competencies. The most relevant discussion and conclusions are based on the importance of reflection as a learning enhancer, the duration and intensity of the interventions and the alignment of all the resources used that interact synergistically around the achievement of the objectives, topics broadly included in the scientific literature.

Keywords: non-formal education; leisure and education; educational innovations; emotional development.
\end{abstract}

\title{
Introducción
}

Hoy en día se acepta indiscutiblemente que las actividades de ocio en el ámbito de la educación no formal impactan educativamente en los niños y en los jóvenes (Macías, 2004), por ello es importante que tanto las familias como los participantes valoren la importancia de utilizar el ocio de manera positiva y activa como un proceso de crecimiento y maduración. La educación no formales un proceso intencional de propuestas educativas cuyas actividades, o bien no responden a los métodos habituales de la educación formal, o bien no pertenecen a la enseñanza reglada y a los requisitos académicos de la misma (Trilla, 1997). No fue hasta bien entrada la democracia española cuando se recuperó la idea de que las actividades de tiempo libre también educan, y se otorgó valor pedagógico a la relación con la naturaleza y al desarrollo físico y mental (Calderón, Gustems y Calderón, 2016).

Las actividades de ocio educativo generan un clima propicio de vivencias, un ambiente lúdico y al aire libre donde se pueden conseguir multitud de objetivos pedagógicos, que en el marco de la enseñanza formal cuestan más de alcanzar. 
En este contexto, destacan los campamentos o colonias vacacionales, y los casales educativos. Los casales se distinguen de los campamentos por no contemplar la pernoctación.

La actividad actual de ambos, casales educativos y campamentos, abarca una amplia oferta; desde los campus deportivos a otras opciones como los campus de idiomas, campamentos urbanos, centros de multiaventura, centros de equitación etc. Entre la gran oferta de propuestas, destacamos el casal emocional, un programa educativamente innovador y que será objeto de estudio en este trabajo.

Un casal es, siguiendo a Mellado y Mellado (2006), una experiencia educativa de primer orden caracterizada por tener una duración relativamente corta, en un ambiente o medio desconocido donde no hay profesores, entendidos de forma tradicional, y donde la familia juega un papel relativamente secundario. No obstante, sus efectos suelen dejar una huella vital que contribuye indiscutiblemente en el proceso madurativo de los participantes.

En este trabajo se presenta la aportación en educación emocional del Método La Granja, aplicado en un casal de verano realizado en la granja escuela de Santa María de Palautordera.

Se trata de la incorporación intencionada de la educación emocional dentro del ámbito de las actividades de ocio infantil y juvenil durante las actividades de verano, lo que puede considerarse un hecho inédito en España.

\section{Las colonias vacacionales: origen y evolución histórica}

El contexto histórico de los campamentos y las colonias en España puede dividirse en tres períodos: a) desde sus orígenes con el Instituto de Libre Enseñanza hasta la Guerra Civil Española, b) durante la dictadura de Franco y c) a partir de la democracia. En cada período, los campamentos han tenido unos valores y unos criterios pedagógicos concretos.

Durante la Revolución Industrial, la población abandonaba el campo para ir a trabajar a las ciudades por lo que aumentaron los suburbios o zonas de concentración de población viviendo en pésimas condiciones. En ese momento y no desvinculado con lo anterior, empezaron a aparecer propuestas y movimientos innovadores sobre educación. Destacaron figuras como las del suizo Pestalozzi y su seguidor Fröbel, quienes difundieron las ideas rousseaunianas sobre educación natural. Para ellos, la naturaleza era una fuente inagotable de recursos para la educación, y facilitadora de la implantación de métodos activos y prácticos. Sus metodologías pedagógicas se basaban en la libertad y en la actividad, otorgando al juego un valor primordial y considerando que el desarrollo físico formaba parte del desarrollo integral de la persona.

En España, esta renovación educativa se incorporó después de la muerte del rey Fernando VII en 1833. Posteriormente, la Revolución de 1868 y el movimiento krausista introducido en España por Julián Sanz del Río, se convirtieron en el motor de la renovación pedagógica (Otero, Navarro y Basanta, 2013). En este contexto surgió en 1876 la Institución de Libre Enseñanza (ILE) de la mano de un grupo de catedráticos despojados de sus cátedras, como Giner, Salmerón o Azcárate. La ILE dio gran importancia a la experimentación y a las excursiones en la naturaleza, a emplear el 
juego como capacitador para la vida real, valoró la educación artística y estética, la coeducación entre familia y escuela, y potenció la formación activa e integral, así como la educación física y moral, siendo uno de los pilares básicos de la renovación educativa en España (Calderón, Gustems y Calderón, 2016, Otero, Navarro, y Basanta, 2013, Rodríguez, 2016).

En 1882, se celebró en España el primer Congreso Nacional Pedagógico impulsado por la ILE, en él se difundió el valor de las excursiones educativas y la importancia de determinadas prácticas físicas e higiénicas, que hasta el momento habían sido exclusivas de la clase burguesa. Poco después, en el año 1887, llegó la primera colonia o campamento escolar de la mano del Museo Pedagógico Nacional liderado por Manuel Bartolomé Cossío, cuyo objetivo era realizar "una acción reparadora sobre los niños necesitados, fortaleciendo su salud física y moral mediante la estancia en plena naturaleza" (Jiménez-Landi, 2005, p. 583). En 1936, como consecuencia de la guerra civil y la posterior dictadura se organizó la última de estas colonias. Tras un paréntesis de 43 años, en 1979, las colonias de verano de la ILE pudieron reanudarse.

Durante la dictadura, es la Iglesia Católica la que se implica en la organización de las colonias o campamentos, ejerciendo una clara misión apostólica. Se crea el Secretariado de Colonias de Cáritas que, en 1960, impartió el primer curso de Monitores de Tiempo Libre y que dio un gran impulso a las parroquias para la organización de sus propias colonias y excursiones (Granero y Lesmes, 2009). Paralelamente, surge la Organización Juvenil Española que contribuye a flexibilizar la organización de las colonias y a la especialización en esta temática (Calderón, Gustems y Calderón, 2016).

En un tercer período, con la instauración de la democracia, las competencias relacionadas con las colonias escolares y de verano, se traspasaron al Departamento de Juventud de cada Comunidad Autónoma. En Cataluña, la Generalitat regula las actividades de ocio infantil y juvenil con la ley de 1981. Conviene tener en cuenta que en esta etapa se fue generalizando el bienestar social, de manera que las colonias vacacionales y escolares perdieron su objetivo higienista, sustituyéndolo por el propósito de mejorar la socialización y disminuir el "ocio pasivo".

A partir de los años 90, la oferta de campamentos y casales de verano es muy amplia y, según Mellado y Mellado (2006, p. 28), los hay educativos y los hay "aparcadero de niños/as más o menos divertidos o estelares donde el reclamo de una serie de actividades o personajes pareciera ser el objetivo". A nuestro entender, para que los campamentos o casales de verano sean educativos, han de perseguir, como mínimo, un objetivo pedagógico, es decir, promover el aprendizaje, como continuación del proceso de desarrollo tanto afectivo como intelectual y motórico de los niños y jóvenes. Estos no dejan de aprender en verano, de hecho, contrariamente, es justo durante estos meses cuando están más despiertos, pues la rutina del curso y la aceleración del día a día desaparecen. Están más abiertos a adquirir aprendizajes que no suelen contemplarse en la enseñanza reglada, en la que todo el tiempo disponible se destina a la adquisición de conocimientos curriculares (Mellado y Mellado, 2006).

Tanto el casal como las colonias se caracterizan por ser una actividad limitada en un corto período, sin seguimiento posterior, aislada de experiencias similares, con talleres inusuales que favorecen el contacto y el intercambio entre los niños y jóvenes, fomentando de manera clara la creatividad, la autonomía, la responsabilidad y la 
diversidad. Asimismo, incorporan todos los recursos didácticos que puedan favorecer una mayor eficacia en la adquisición de los objetivos propuestos (desde el material pedagógico hasta el bosque o los animales, actividades como recoger la mesa, hacerse la cama o ir a la piscina).

Actualmente las colonias o campamentos conforman la base del ocio educativo de la educación no-formal. De acuerdo con Macias (2001, p.604) “su papel fundamental es contribuir a aprender a vivir, a conocer y experimentar a través del medio para que sean útiles para comprender e integrase de manera satisfactoria en la sociedad". En realidad, la educación formal y la educación no formalcomparten la misma finalidad que no es otra que contribuir al desarrollo integral de los niños y jóvenes.

\section{El casal emocional de La Granja}

La Granja, granja escuela de Sta. Maria de Palautodera fue fundada en 1984 por la familia Gutiérrez-Lestón, como un espacio pedagógico que ofrecía un servicio que las escuelas empezaban a necesitar: un lugar seguro, adecuado y moderno para aproximar a los escolares a la naturaleza, al medio ambiente y a los animales de granja desde la vivencia y la experimentación directa. Coincidió con una nueva corriente educativa tras el franquismo, que volvía a dar valor al aprendizaje vivencial, base del ocio educativo y de la formación no-reglada que, como se ha expuesto anteriormente, ya tenía una historia que respaldaba sus beneficios.

La Granja se convirtió en un centro de referencia que contaba con una programación pedagógica adaptada a todas las etapas educativas (de 3 a 17 años). El hecho de que la familia Gutiérrez-Lestón hubiera vivido durante 12 años en Hamburgo (Alemania), fue determinante para ofrecer un servicio avanzado en la España de la época, muy profesionalizado y totalmente orientado a dar respuesta a las necesidades del profesorado y sus alumnos. Prueba de ello fue la alta ocupación de sus instalaciones desde el primer momento, llegando a atender anualmente a más de 12.000 alumnos de escuelas públicas y privadas, con actividades como colonias y excursiones escolares durante el curso, los tradicionales campamentos de verano desde 1987, o el casal de verano, a partir de 1998.

Sin embargo, entre los años 2002 y 2004 se detectó que todo aquello que les había funcionado durante 20 años perdía consistencia. Los alumnos ya no escuchaban como antes y ante un conflicto no conseguían modular actitudes ni comportamientos. La sorpresa primero, el desencanto y la frustración después, aparecieron en el equipo de educadores. La reflexión sobre esta nueva situación permitió llegar a la conclusión de que era necesario hacer algo diferente, ya que los niños y jóvenes habían cambiado; presentaban fuertes carencias emocionales tales como miedos, baja autoestima, aceleración, dependencia, inseguridad y desconfianza, así como actitudes egoístas, ansiedad y baja tolerancia a la frustración, como si se hubiera sustituido la cultura del ser por la cultura del tener (Gutiérrez-Lestón, 2014).

Como respuesta a esta situación, el equipo de educadores empezó a investigar y formarse en nuevos métodos y maneras de educar, descubriendo, en la educación emocional, la herramienta con la que mejores resultados se obtenían (Keefer, Parker y Saklofske, 2018). Los frutos empezaron a aparecer tímidamente durante el año 2008, 
con una mejora en la escucha y en las actitudes de los chicos. A partir de los avances observados, se formó tanto al equipo de educadores como al staff, reorientando las actividades y diseñando nuevos talleres con el objetivo de fomentar el desarrollo de competencias emocionales. Así, se reinventó el concepto de La Granja, con una nueva mirada que permitió en 2011 que el casal de verano, se transformase en casal emocional.

Este casal utiliza su propia metodología, "El Método La Granja”, el cual se ha desarrollado para tal fin (Figura 1), y que tiene el propósito de educar mediante el desarrollo de las competencias emocionales a niños y adultos. Se basa en el marco teórico de Bisquerra y Pérez (2007) y utiliza la metodología Outdoor Training (Silva, Franco, Nobrega, y Porto, 2014).

El casal emocional trata de fomentar un ocio educativo procurando que los niños se olviden de sus televisores, consolas y ordenadores, y se acerquen a la naturaleza para realizar actividades lúdico-educativas en las que prevalece el desarrollo de valores como la convivencia, la tolerancia, la flexibilidad y el respeto, además de divertirse aprendiendo, mientras fomentan la autonomía y las habilidades sociales y personales. Añadir a estos objetivos la creciente demanda durante los últimos años, de estimular la espontaneidad de los alumnos como respuesta a la curiosidad y a la necesidad de mejorar la creatividad y automotivación de los chicos, junto con la necesidad de relajación para aumentar el sentimiento de felicidad. Paralelamente la oferta del casal permite a muchas familias conciliar la vida laboral, ya que empieza la semana siguiente de finalizar el curso escolar, en horario de $9 \mathrm{~h}$ a 17,30h de lunes a viernes durante 5 semanas en verano. Se distingue así de las colonias vacacionales en que los participantes no son aislados de sus familias más que unas horas al día, ya que pernoctan en sus hogares, donde pasan también el fin de semana.

De acuerdo con el marco teórico de referencia, se entiende la competencia emocional como "el conjunto de conocimientos, capacidades, habilidades y actitudes necesarias para comprender, expresar y regular de forma apropiada los fenómenos emocionales" (Bisquerra y Pérez, 2007, p. 69). Para desarrollar la competencia emocional será necesario atender las cinco dimensiones del modelo: consciencia emocional, regulación emocional, autonomía emocional, competencias sociales y competencias para la vida y el bienestar.

El casal emocional se centra en el desarrollo de estas competencias, entendiendo, al igual que Durlak, Domitrovich, Weissberg y Gullotta,(2015), que ello favorece el afrontamiento de las circunstancias de vida con mayores probabilidades de éxito, y que su dominio mejora los procesos de aprendizaje, las relaciones interpersonales, la solución de problemas y el bienestar personal. Todo ello en un marco potenciador, el de la educación no formal y concretamente el ocio formativo (Baker, 2018), donde se acelera el aprendizaje vital de una manera difícil de conseguir en el contexto del sistema educativo actual (tal y como hoy en día se orienta en la mayoría de los centros educativos de España).

"El Método La Granja" se basa en el lanzamiento de retos a los participantes con la intención de emocionarlos, ya que la emoción es necesaria para la interiorización de los aprendizajes. Además, se utilizan estímulos positivos de manera continua para provocar la alegría, la cual ayuda a activar a los chicos, incluso a los más apáticos. De hecho, sentirse optimista y potente para afrontar los desafíos diarios es una competencia socioemocional reconocida por Payton, et al. (2000). En este sentido, hacemos 


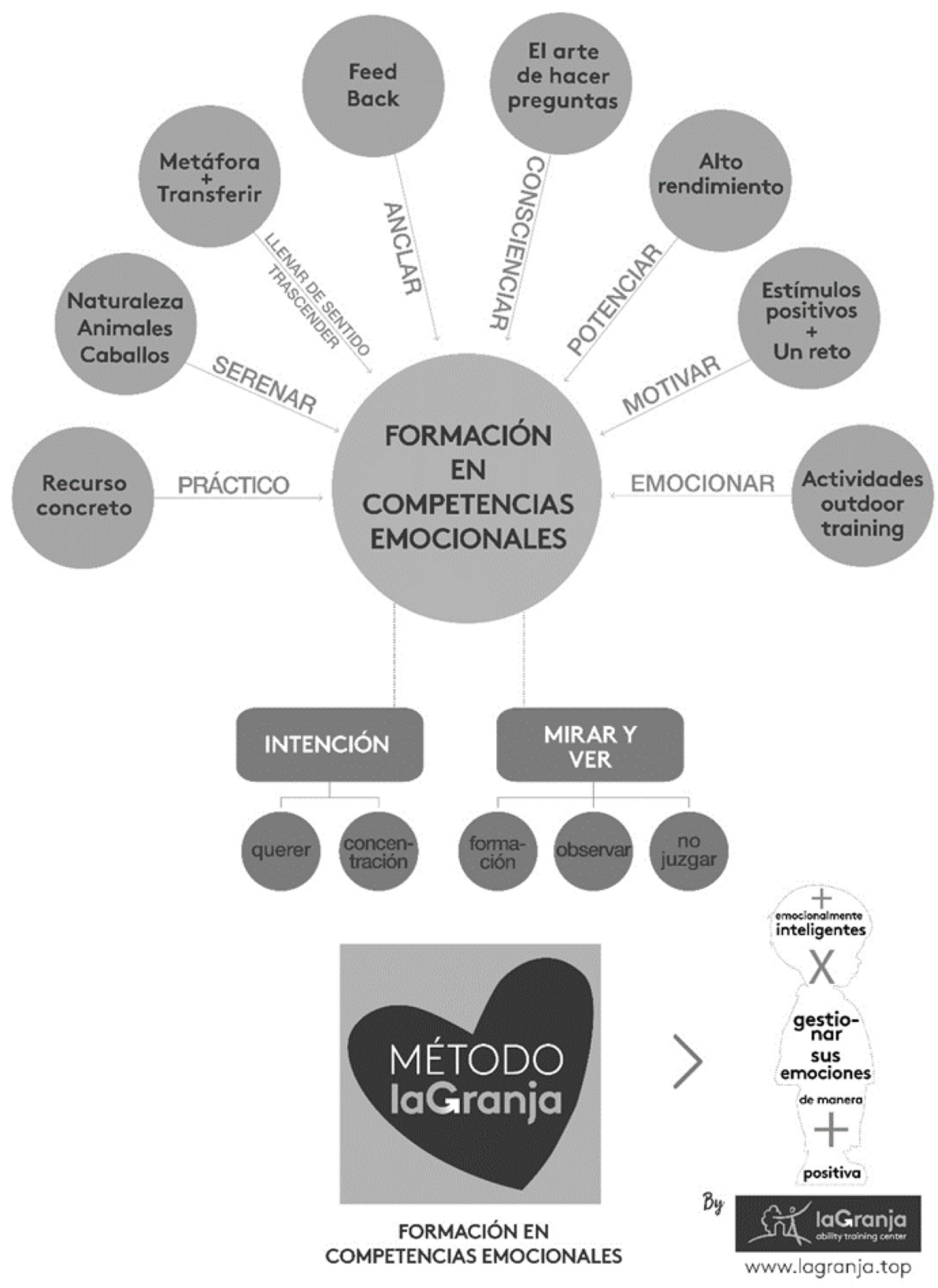

Figura 1. Método La Granja. Versión 2017. 
nuestra la propuesta de Saarni (2000) cuando sostiene la importancia de enseñar a los jóvenes a decidir cómo se quieren sentir, entendiendo que conseguirlo es el máximo grado de autoeficacia emocional.

El Método La Granja utiliza el alto rendimiento, con actividades cortas que evitan el aburrimiento o la repetición de conceptos y potencian el resultado. Para ello los educadores han sido formados en una actitud de concentración y atención plena para aprovechar todo aquello que pasa y todo aquello que no se produce o falta durante una dinámica, potenciando así el aprendizaje y obteniendo el máximo rendimiento de cada minuto a pesar del escaso tiempo (cinco semanas) de duración del casal.

Como complemento, las herramientas del coaching, como el arte de hacer preguntas y el feedback, permiten que los niños sean los verdaderos protagonistas no solo de la actividad práctica, sino también y sobre todo de las reflexiones.

La metáfora también forma parte del Método La Granja. Esta permite trabajar desde el inconsciente de cada participante, facilitando el entendimiento y la transferencia de aquello que pasa en la actividad a diversos aspectos de su vida familiar o de la escuela. Así se consigue ampliar y trascender el aprendizaje encontrándole un sentido resonante más sostenible en el tiempo.

A todo esto, hay que añadir la naturaleza, el bosque, los caballos de doma natural o los animales de granja, elementos que también contribuyen al Método La Granja pues ejercen un efecto relajante en los niños, imprescindible en estos últimos años en los que se observa una gran aceleración en ellos. Esta mayor sensación de tranquilidad favorece el autoconocimiento y, al "darse cuenta", se posibilita avanzar y obtener resultados en el desarrollo de la competencia emocional de cada uno de ellos.

El casal emocional, por extensión, tiene plantea el objetivo de trabajar no solo con los niños, sino también con sus padres, pues el contacto diario cuando los traen y recogen se convierte en una oportunidad para provocar cambios más sostenibles en el tiempo. Además, cada semana los padres leen con sus hijos dos capítulos del cuento que cada verano constituye el hilo conductor del casal.

Tabla 1

Sintesis del programa del casal emocional

\section{Casal emocional}

\begin{tabular}{ll}
\hline Propósito & $\begin{array}{l}\text { Fomentar el desarrollo de las competencias emocionales de los } \\
\text { participantes. }\end{array}$ \\
\hline Objetivos & -Afianzar la confianza personal en base al descubrimiento de sus \\
específicos & talentos y habilidades. \\
& -Comprender el valor del otro estableciendo relaciones interpersonales \\
& sanas, basadas en el respeto y la solidaridad. \\
& -Experimentar la autonomía personal en la ejecución de distintas \\
& rutinas y actividades. \\
& -Descubrir en la naturaleza y las actividades en equipo una fuente de \\
& disfrute y aprendizaje. \\
\hline
\end{tabular}




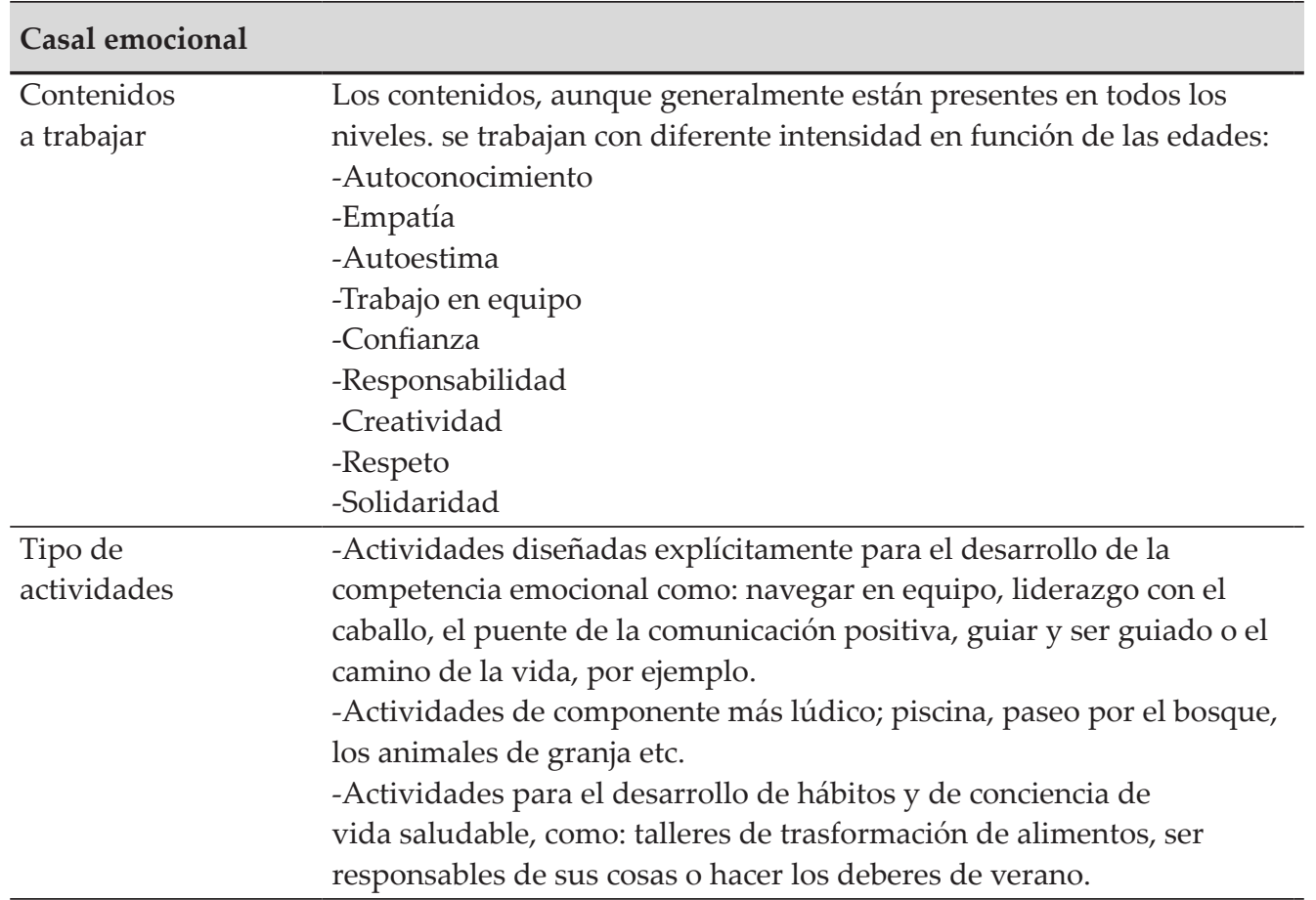

\section{Método}

\section{Objetivos}

La investigación que se presenta se basa en la evaluación de los resultados del casal emocional del verano de 2017 realizado en "La Granja". Concretamente, el objetivo es valorar el efecto de la participación de los alumnos entre 8 y 14 años en términos de mejora de sus competencias emocionales, así como su percepción sobre la utilidad de lo aprendido.

Al tratarse de una evaluación, sigue un paradigma pragmático (Mertens, 2015; Karr y Kemmis, 1983) en el que se combinan datos obtenidos a través de distintos instrumentos de recogida de información, que responden a su vez a diferentes lógicas de investigación, inductiva y deductiva. En él, se incluye la aplicación del Cuestionario de Desarrollo Emocional (CDE) a los niños, antes y al final de su participación en el casal de verano de La Granja. Además, al finalizar el casal se preguntó a los niños sobre los aprendizajes realizados para obtener la riqueza de la exploración de significados personales respecto a la experiencia. 


\section{Población y Muestra}

Presentamos aquí los resultados de los 127 niños y niñas de la muestra que se corresponden con los participantes de las cinco semanas en el casal emocional y que cumplimentaron todos los cuestionarios. De estos, el 53.5\% son niños y el $46.5 \%$ niñas.

Los participantes no fueron escogidos al azar, sino que son los inscritos en el casal del verano del 2017.

\section{Procedimiento de recogida y análisis de datos}

Todos los datos son tratados con confidencialidad y sujetos a los estándares éticos requeridos en el trabajo con seres humanos. Todas las familias han sido informadas y han tenido derecho a ejercer la oposición a la participación de sus hijos o hijas en la investigación. Todos los datos cuantitativos se han tratado utilizando el procesador estadístico SPSS v.21. Por su parte, los datos cualitativos han sido trabajados a nivel lexicométrico, mediante el programa informático Iramuteq y además se ha hecho un análisis clásico del discurso basado en el significado manifiesto de las respuestas, utilizando el modelo de Miles y Huberman (1984).

Al principio del casal emocional se aplicó a los niños el CDE para evaluar la competencia emocional. Participaron durante cinco semanas en el casal emocional, y tras ese período, los niños respondieron de nuevo a esta prueba junto a los cuestionarios cualitativos elaborados ad hoc para esta investigación.

\section{Instrumentos}

La evaluación de la competencia emocional se ha realizado con las versiones para niños de 9-13 años y de adolescentes del cuestionario de desarrollo emocional CDE (CDE-9-13 y CDE-SEC, respectivamente). Están diseñados de acuerdo con el modelo de competencia emocional del GROP (Grupo de Investigación en Orientación Psicopedagógica). Consiste en una escala de autoinforme con ítems con formato de respuesta tipo Likert, con 11 opciones de respuesta en una escala de 0 (completamente en desacuerdo) a 10 (completamente de acuerdo). Los resultados que se obtienen permiten detectar las necesidades que cada alumno puede tener a nivel global y en cada una de las dimensiones. Los estudios realizados con muestras de diversas edades indican que su índice de consistencia interna medida mediante el coeficiente alfa de Cronbach, oscila según los casos entre .79 y .85. (Pérez-Escoda, 2016)

Al final del postest, se añadieron tres preguntas abiertas relativas a: los aprendizajes autopercibidos por cada niño o niña y su utilidad futura a fin de valorar su percepción.

\section{Análisis de datos}

Para medir los cambios en la competencia emocional de los participantes se ha procedido a aplicar pruebas estadísticas de contraste de medias entre los resultados obtenidos en el CDE en la situación inicial y final. 
Inicialmente se comprobaron los coeficientes de Kolmogorov-Smirnov y Levene como indicadores de normalidad y homocedasticidad previos a la aplicación de t-Student para el contraste de muestras relacionadas.

Las respuestas abiertas se han procesado, por una parte, a través de un análisis exploratorio lexicométrico, y por otra, se ha efectuado un análisis del discurso, sobre el contenido manifiesto en las respuestas. Para ello, una vez recogida la información, se inicia el proceso con la reducción de datos, se extraen las respuestas a las preguntas abiertas y se agregan ciertos datos sociodemográficos del grupo de informantes y que nos parecen relevantes para las conclusiones. Una vez hecho esto, se inicia la fase de generación de categorías, que se ha abordado a través de un proceso iterativo de revisión, para concluir con la fase de extracción de conclusiones. La primera categorización resultó muy cercana a las dimensiones planteadas en el modelo de educación emocional de Bisquerra y Pérez (2007), 1 Conciencia emocional, 2 Regulación emocional, 3 Autonomía emocional, 4 Competencia social y 5 Competencias para la vida y el bienestar.

Esto nos llevó a categorizar nuevamente, pero en esta ocasión, identificando la competencia emocional que se hallaba tras la respuesta. Esto se hizo con una estrategia de triangulación entre cuatro observadoras, así, cada investigadora hizo su propia categorización que luego fue consensuada.

\section{Resultados}

\section{Evolución de las competencias emocionales según $\mathrm{CDE}$}

Tras obtener un ( $p>$.05) en todas las variables en la prueba Kolmogorov-Smirnov y Levene se procedió a aplicar la prueba $\mathrm{T}$ de muestras relacionadas entre la situación inicial y final.

Como se observa en la Tabla 2. tanto en la competencia emocional total como en sus cinco dimensiones: conciencia, regulación, autonomía, competencias sociales y competencias de vida y bienestar, las puntuaciones en la fase postest, después de su participación, han aumentado. No obstante, conviene observar los resultados del contraste de medias para valorar si estas diferencias son significativas.

Tabla 2

Evolución competencias emocionales pre-post (CDE)

\begin{tabular}{lccccc}
\hline & Media & DT. & t & Sig. & d Cohen \\
\cline { 2 - 6 } Pre_Total_C.Emocinal & 6.73 & 0.99 & -5.03 & 0.000 & -0.33 \\
Postest_Total_C.Emocinal & 7.07 & 1.07 & & & \multirow{2}{*}{-0.18} \\
\hline Pre_Consciencia & 7.46 & 1.36 & -2.76 & 0.007 & \multirow{2}{*}{-0.40} \\
Postest_Consciencia & 7.71 & 1.35 & & & \multirow{2}{*}{0.000} \\
\hline Pre-Regulación & 5.69 & 1.43 & -5.05 & & \\
Postest_Regulación & 6.25 & 1.35 & &
\end{tabular}




\begin{tabular}{lccccc}
\hline & Media & DT. & t & Sig. & d Cohen \\
\cline { 2 - 6 } Pre-Autonomía & 6.60 & 1.37 & -3.98 & 0.000 & -0.30 \\
Postest_Autonomía & 7.01 & 1.35 & & & \multirow{2}{*}{-0.18} \\
\hline Pre_C.Social & 6.29 & 1.64 & -2.48 & 0.014 & \multirow{2}{*}{0.08} \\
Postest_C.Social & 6.60 & 1.75 & & & \multirow{2}{*}{0.257} \\
\hline Pre_C.Vida_y_Bienestar & 7.52 & 1.20 & -1.14 & & \\
Postest_C.Vida_y_Bienestar & 7.63 & 1.40 & & & \\
\hline
\end{tabular}

$\mathrm{N}=127$

La diferencia en la competencia emocional total se produce con un nivel de significación de .000 a favor de la situación final, pudiendose afirmar que hay mejoras entre el antes y el después del casal emocional de La Granja.

Del analisis de las diferentes dimensiones evaluadas, se concluye que en todos los casos, menos en competencias de vida y bienestar, los cambios apreciados son estadísticamente significativos con una probabilidad entre $p=.000$ y $p<.014$ y también a favor de la situación final, indicando una mejora en el nivel de desarrollo de estas competencias. Para la dimensión competencias de vida y bienestar, las diferencias no son estadísticamente significativas, por lo tanto no se estima que se hayan producido cambios en esta dimensión.

Además del contraste de medias se ha procedido a calcular la magnitud de los cambios, para ello se ha estudiado la $d$ de Cohen (vid Tabla 2). A partir de los resultados se detectan cambios muy similares a los habituales como consecuencia de procesos formativos (Rosenthal, 1996), con magnitud alrededor de 30 en la competencia emocional total, y en las dimensiones de regulación y la autonomía emocional. En las dimensiones consciencia y competencia social los cambios son muy pequeños.

\section{Percepción de los aprendizajes realizados}

Como resultado del análisis lexicométrico, el gráfico de la Figura 2 ofrece una visión general del discurso y muestra los resultados obtenidos, que resultan especialmente resonantes. Como puede apreciarse, los términos utilizados con mayor frecuencia por el grupo en su totalidad y que se representan como nodos de significado, son: hacer, aprender y amigo, seguidos de otros enlaces en cuyo centro se hallan las palabras: bueno, diversión, persona, cosa, útil, gente, respetar y conocer.

En el análisis particular de cada uno de estos nodos, se observan interesantes coincidencias o coocurrencias que resultan significativas en el análisis que nos ocupa, incluso en las más alejadas del centro del gráfico. Encontramos, por ejemplo: gente, asociado a convivir, conocer. Otra cadena de palabras, asociadas en este caso a "miedo" son: enfrentar, superar, dar, opinión;"útil“" que aparece al lado de empatia, considerar y alegría. Todas ellas con un alto contenido emocional y relacionado con el programa ofrecido. 


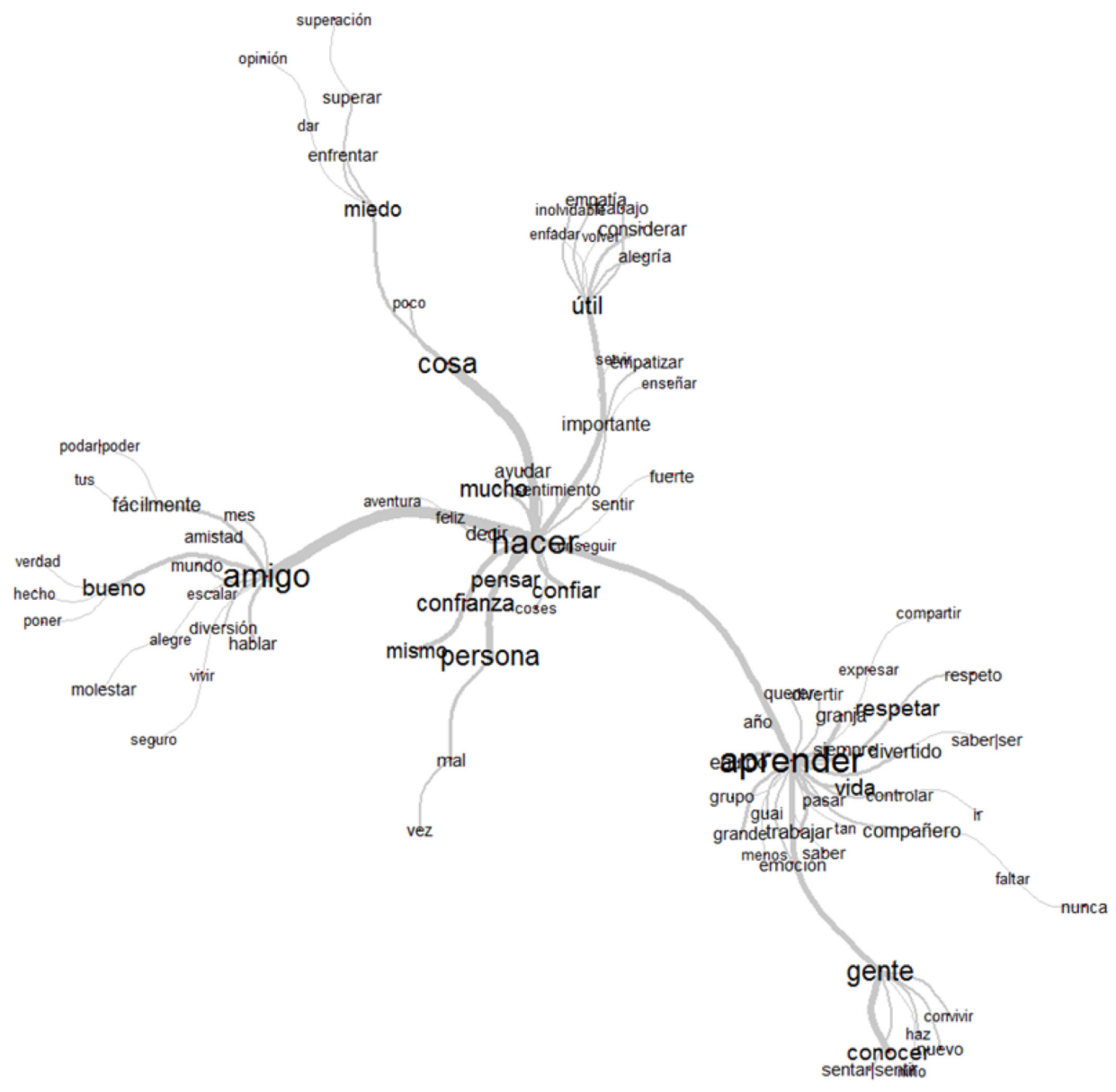

Figura 2. Gráfico del análisis de similitudes de las respuestas a las preguntas abiertas

Hay otro gran nodo en la palabra "aprender" rodeada de otras como: vida, divertido, granja, emoción, respetar, trabajar y compañero. Todas ellas parece que aluden a la forma propuesta para lograr el aprendizaje, aspectos que se trabajan intencionadamente en el método del casal emocional de la Granja.

Otro nodo central surge alrededor del término "amigo", esta palabra se vincula principalmente con bueno, fácilmente, diversión y hablar y es que, frecuentemente, se alude a conocer nuevas personas, hacer amigos, ampliar las relaciones afectivas. No 
deja de ser interesante la analogía entre esta comunidad de términos y la experiencia vivida en el casal emocional, en el contacto con el bosque, con los animales y especialmente con otros en una forma de relación diferente a la que habitualmente tienen y que constituyen una necesidad real durante la infancia y adolescencia.

El tercer y último núcleo es "hacer". Esto se vincula, de nuevo, con la experiencia, el hacer de forma autónoma y personal, lo que se confirma con algunos de los términos inmediatos como uno mismo, persona, decir, feliz, aventura, mucho e importante. También hay una serie de términos asociados como: pensar, confiar, sentimiento o ayudar y que parecen conectar con un hacer menos activo y más reflexivo.

Tras el análisis lexicométrico, y como se ha descrito en el método, después de asociar el conjunto de categorías emergentes a las competencias emocionales del modelo teórico, se ha realizado un análisis descriptivo de los hallazgos, contabilizando la cantidad de fragmentos de texto que se corresponden con el desarrollo de cada una de las competencias emocionales.

Así, como se aprecia en la Figura 3, la competencia social es la que más se desarrolla en la totalidad del grupo, seguida de la regulación emocional, la autonomía, la conciencia y, por último, las competencias para la vida y el bienestar. Merece una mención especial el hecho de que estos resultados se asemejan a los hallados con la medición del cuestionario CDE.

Ya en el análisis lexicométrico exploratorio, se observó la posible incidencia de la variable sexo en la percepción de utilidad de lo aprendido y el desarrollo de las competencias emocionales, por lo que se ha querido profundizar. Para establecer las diferencias existentes por sexo, se calculó el porcentaje de chicas/chicos que habían mostrado en sus respuestas las diferentes dimensiones de la competencia emocional, considerando que la totalidad de respuestas del grupo (chicas y chicos) para cada competencia emocional era del 100\%.

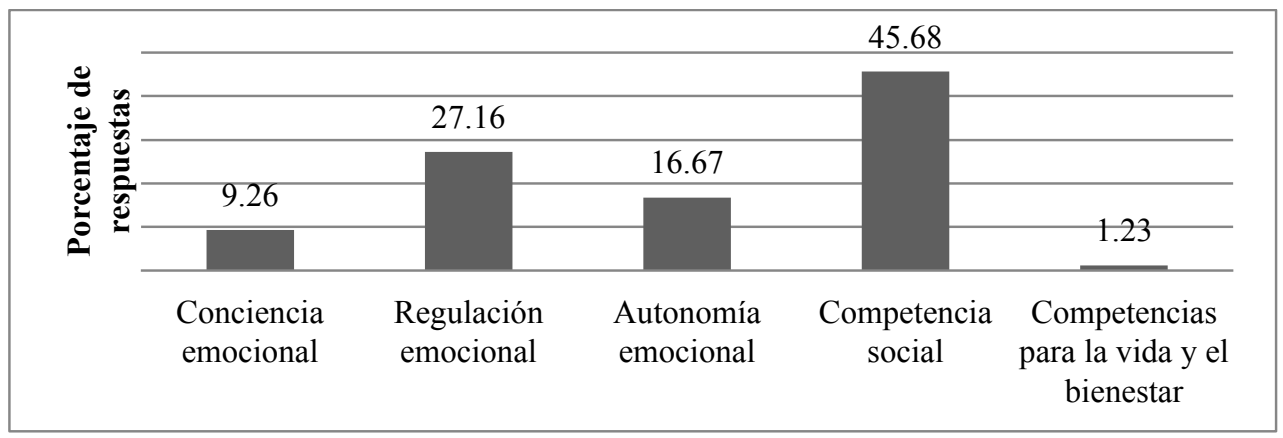

Figura 3. Porcentaje de respuestas categorizadas según las áreas de competencias emocionales, grupo total.

Como puede apreciarse en el gráfico, parece que las chicas hacen referencia en sus respuestas a dimensiones internas, intrapersonales: conciencia, regulación y autonomía emocional. Mientras que los chicos destacan más las competencias de relación o interpersonales: competencia social y competencias para la vida y el bienestar. 


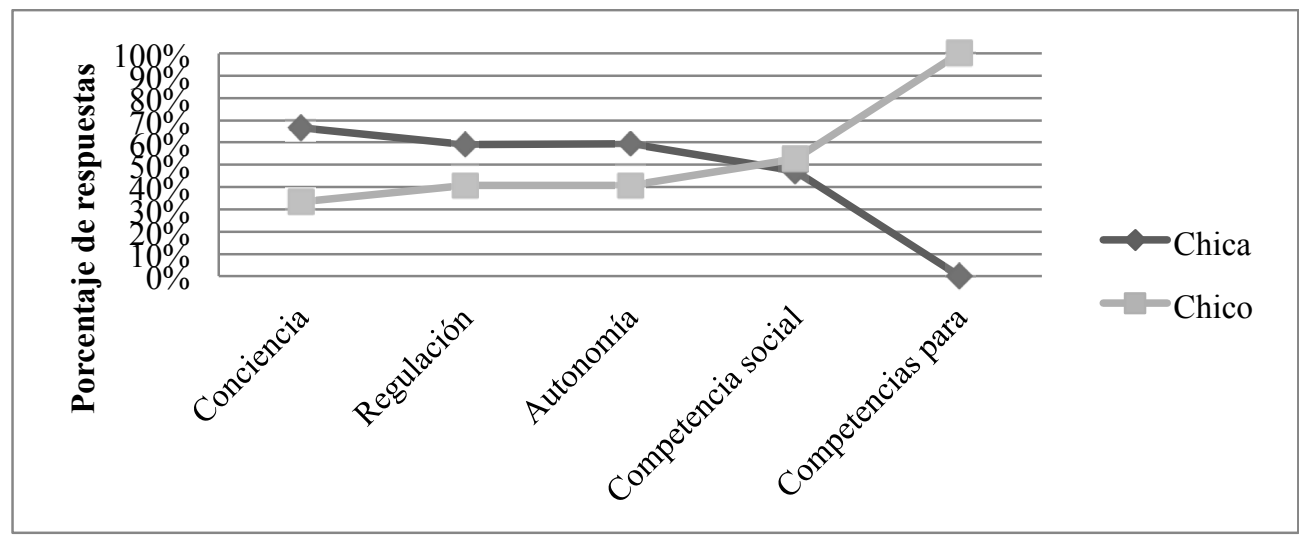

Figura 4. Porcentaje de respuestas categorizadas por dimensiones de la competencia emocional, en función del sexo.

Otro elemento de comparación dentro del grupo total, es el programa que siguieron durante su período en el casal emocional. Los programas se diferencian en cuanto a grupo etario, de modo que las actividades ejecutadas con los grupos de participantes que pertenecen a primaria Ciclo Medio, primaria Ciclo Superior y ESO, varían entre ellos. Para el análisis se procedió de manera similar a la variable sexo, se calculó el porcentaje de fragmentos correspondientes a las diferentes dimensiones de la competencia emocional en cada uno de los tres grupos.

Como puede observarse en la Figura 5, la competencia social, nuevamente aparece como la más repetida por los tres grupos, destaca especialmente el grupo de Ciclo superior.

Por último, se ha hecho una breve recopilación de las respuestas más elocuentes referidas a cada una de las categorías.

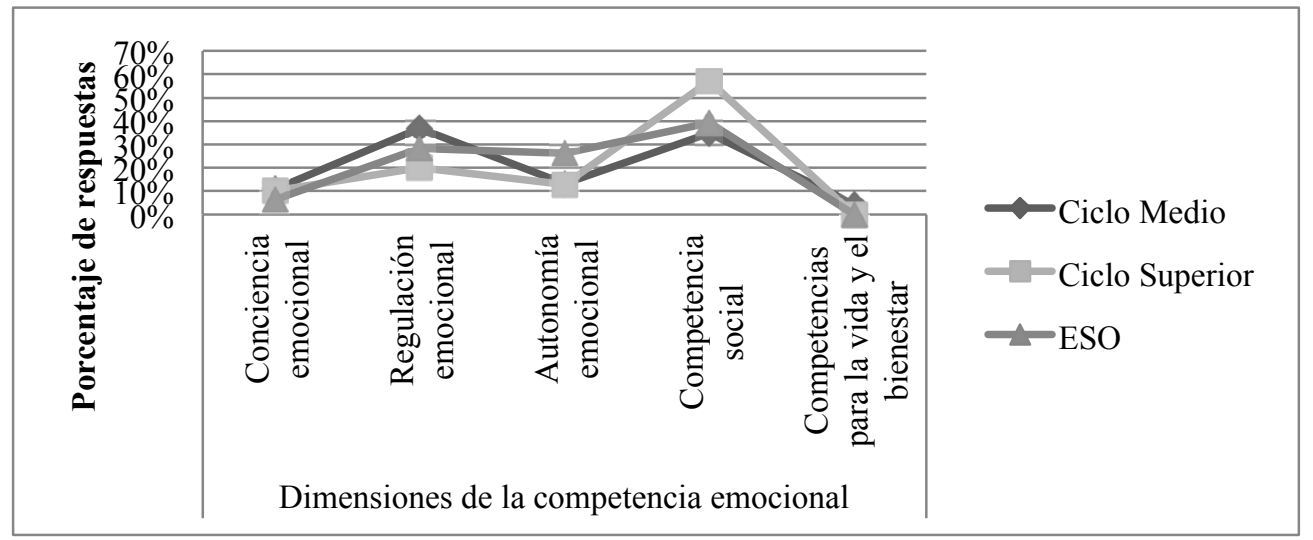

Figura 5. Porcentaje de respuestas categorizadas por dimensiones de la competencia emocional, en función de los grupos según ciclo. 
Conciencia emocional. Se refiere a la toma de conciencia de las propias emociones y las de los demás. Se aprecia en los siguientes comentarios:

"He aprendido muchas cosas, pero la que me será útil es que los miedos se tienen que superar poco a poco". (Chica, 11)

"He aprendido a saber las emociones que siento y escoger los amigos de verdad". (Chico, 13)

Regulación emocional. Se refiere a una gestión apropiada de las emociones y a disponer de recursos para el afrontamiento de los retos cotidianos.

"He aprendido a confiar en los demás y en mi misma, a hacer las cosas en grupo cooperando, también a compartir y a expresar mis sentimientos". (Chica, 8)

"He aprendido a perdonarme a mi mismo". (Chico, 9)

Autonomía emocional. Incluye la autogestión personal, la autoestima, autoconfianza, autoeficacia, responsabilidad y actitud positiva. La capacidad para autogenerar emociones apropiadas en distintas situaciones, dando paso al bienestar subjetivo.

"En La Granja he aprendido muchas cosas como ser autónoma, perder el miedo a la hora de hacer cosas nuevas y con gente nueva. Pero sobre todo he aprendido a dar una oportunidad a la gente que no conozco". (Chica, 12)

"También he aprendido que siempre tienes que dar tu opinión, porque yo, a veces, no doy mi opinión cuando tenemos que decidir alguna cosa por miedo de que los otros piensen mal de mí, pero me han enseñado que todas las personas en un grupo son muy importantes y que sus opiniones también lo son" (Chica, 12).

Competencia social. Se refiere al uso adecuado de los recursos personales frente a las relaciones sociales. Implica el dominio de la comunicación asertiva, la cooperación, el respeto por los demás.

"A respetar a los demás, a no molestar a los demás cuando están enfadados". (Chico, 10) "He aprendido a relacionarme mejor con personas que no conocía antes, también he aprendido a hacer más cosas en grupo [...], así que he vivido experiencias nuevas". (Chico, 13)

Competencias para la vida y el bienestar. Implica el afrontamiento de las distintas situaciones de vida, de manera equilibrada y responsable. Desde el planteamiento de objetivos de vida asumibles, la actuación y toma de decisiones ética y responsable.

"He aprendido a vivir la vida con más optimismo, menos perfeccionismo y también a disfrutarla más". (Chico, 9) 


\section{Discusión y conclusiones}

En este trabajo, se aportan evidencias del efecto de la participación en el casal emocional de La Granja de los niños entre 8 y 14 años, en la mejora de sus competencias emocionales, así como su percepción sobre la utilidad de lo aprendido.

El contexto es un casal que introduce la educación emocional como objetivo de su actuación, para lo cual ha desarrollado un método propio que aparece como una respuesta innovadora frente a las nuevas necesidades educativas de los niños y niñas de hoy en día.

Los resultados observados permiten señalar que, efectivamente, se ha producido una mejora de la competencia emocional, especialmente en regulación $(d=.40)$ y autonomía emocional $(d=.30)$, seguido de la competencia social $(d=.18)$.

Los datos cualitativos también apuntan en esta dirección. Destaca un mayor número de comentarios referidos a la competencia social, seguidos de aquellos que refieren a aprendizajes relacionados con la regulación y con el desarrollo de su autonomía emocional. Estos resultados son congruentes con los observados por Flynn, Ricker, Dolezal, Kunin y Mellins (2019) en un campamento de verano desarrollado en Estados Unidos.

Igualmente, el análisis lexicométrico destaca los términos "gente", "hacer" y "aprender". El término "gente" puede vincularse con la competencia social, mientras que el "hacer" se vincula con la experiencia personal y autónoma de la confianza en sí mismo, asociado con el desarrollo de la autonomía emocional. Y, finalmente, "el aprender", en un lugar central, puede interpretarse como evidencia de que los participantes perciben una gran utilidad en su paso por el casal. En este sentido, conviene de nuevo rescatar la idea del ocio como estrategia educativa además de lúdica.

Para ello el Método La Granja se basa en actividades vivenciales, altamente motivantes, retos continuos y provocadores que pretenden la atención permanente en el aquí y el ahora. El tipo de comunicación que se establece se basa en escuchar a los participantes, no dar instrucciones, y estas se reemplazan por preguntas que permiten tomar consciencia de lo que sienten y de cómo actuar. Asimismo, la actividad va seguida de un feedback reflexivo que permite aprender desde la emoción y la experimentación para incorporarlo y transferirlo a su vida cotidiana (Schön, 1998).

Dieskstra (2008), en su revisión de diversos metaanálisis sobre los efectos de la educación emocional y social para niños y jóvenes, destacó como una de las variables importantes, para la consecución de buenos resultados, la duración e intensidad de las intervenciones. Destaca que, para ser eficaces en el ámbito escolar, deben tener una determinada duración estimada entre 3 y 6 meses (clases semanales). En nuestro estudio la duración es menor (5 semanas), pero la intensidad es mucho mayor (8 horas diarias), lo que, sumándose al Método La Granja, actúa sinérgicamente y provoca el desarrollo de las competencias emocionales de manera acelerada.

Otro elemento favorecedor de los aprendizajes es acción sistémica: se ofrece formación en su metodología de todo el personal de La Granja, de forma que todo el equipo tiene la intención de interactuar con los niños de forma alineada. Este aspecto también está en concordancia con las recomendaciones sobre la necesidad de la formación previa de los educadores encargados de la implantación de programas de educación emocional (Durlak, Domitrovich, Weissberg y Gullotta, 2015; Keefer, Parker y Saklofske, 
2018; Pérez-Escoda, Berlanga y Alegre, 2019; Torrijos, Hernández y Rodríguez, 2016). Asimismo, es preciso considerar que las familias ya poseían una sensibilidad especial de partida, $y$, además, recibieron indicaciones durante en el casal, de tal manera que se añade la sinergia proveniente del núcleo familiar.

En resumen, en este trabajo se han presentado las aportaciones en educación emocional del Método La Granja con una experiencia innovadora en el ocio educativo, basada en la implantación de la educación emocional en el ámbito no-formal, concretamente en un casal de verano para niños y adolescentes. Se ha demostrado que la experiencia incide en el desarrollo de la competencia emocional de los participantes. Con ello, cabe esperar que, en breve, los centros educativos se animaran a incorporar la dimensión emocional en las actividades de ocio educativo que realizan con sus alumnos.

Para futuros estudios relacionados con el desarrollo de las competencias emocionales, sería interesante ampliar la investigación sobre la sostenibilidad en el tiempo de las competencias aprendidas vivencialmente, y cómo ello ayuda a desarrollar la inteligencia emocional de los niños y jóvenes.

\section{Referencias}

Baker; M. (2018). Welcome to the Bubble: Experiences of Liminality and Communitas among Summer Camp Counsellors. Journal of Youth Development.13 (1-2),24-43 doi: https://doi.org/10.5195/jyd.2018.565

Bisquerra, R. y Pérez, N. (2007). Las competencias emocionales. Educación XXI, 10, 61-82.

Calderón, D., Gustems, J., y Calderón, C. (2016). Objetivos pedagógicos de las colonias y campamentos de verano: una revisión histórica. RES, Revista de educación social. 22, 331-347.

Durlak, J. A., Domitrovich, C. E., Weissberg, R. P., y Gullotta, T. P. (2015). Handbook of Social and Emotional Learning. Research and Practice. Nueva York: The Guilford Press.

Granero, C., y Lesmes, J. C. (2009). Los campamentos de verano como modelo de actividades de tiempo libre juvenil. Madrid: Injuve.

Gutiérrez-Lestón, C. (2014): Palabras de Niño. Santa Maria de Palautordera: Plataforma Editorial.

Flynn, R. M., Ricker, A. A., Dolezal, C., Kunin, M., y Mellins, C. A. (2019). Residential summer camp for youth with special needs: A longitudinal approach to investigating differences in social skills. Children and Youth Services Review, 96, 354-363. doi: https://doi.org/10.1016/j.childyouth.2018.10.036

Keefer, K. V., Parker, J., y Saklofske, D. H. (Eds.) (2018). Emotional Intelligence in Education. Integrating Research with Practice. Cham (Suiza): Springer.

Jiménez-Landi, T. (2005). Las colonias de vacaciones de la Institución Libre de Enseñanza. En P. Dávila, y L.M. Naya (Coords.): La infancia en la historia: espacios y representaciones, 1, 583-594. San Sebastián: Erein

Macías, E. (2004). Aproximación a la intervención de calidad en la educación no formal. Funciones del pedagogo. Revista Complutense de Educación, 15(2), 561-596.

Mellado, A., y Mellado, J.L. (2006) El campamento como medio educativo. Revista de Estudios de juventud, 72, 25-37. 
Mertens, D. (2015) Researched and Evaluation in education and Psychology. $4^{\mathrm{a}}$ Ed.California: Sage

Miles, M., y Huberman, A.M. (1984). Qualitative data analysis. A source book of new methods. Beverly Hills: Sage.

Otero, E.; Navarro, R., y Basanta, S., (2013). Las colonias escolares de vacaciones y la Institución de Libre Enseñanza. Historia y actualidad. Revista de Investigación en Educación, 11(2), 140-1.

Payton, J. W., Wardlaw, D. M., Graczyk, P. A., Bloodworth, M. A., Tompsett, C. J., y Weissberg, R. P. (2000). Social and emotional learning: A framework for promoting mental health and reducing risk behaviors in children and youth. Journal of School Health, 70, 179-185. doi: https://doi.org/10.1111/j.1746-1561.2000.tb06468.x

Pérez-Escoda, N. (2016). Cuestionarios del GROP para la evaluación de la competencia emocional (CDE). En: J. L. Soler, L. Aparicio, J. Díaz, E. Escolano, y M.A. Rodríguez, Inteligencia emocional y bienestar II. Reflexiones, experiencias profesionales e investigaciones (pp. 690-705). Zaragoza: Ediciones Universidad San Jorge. En: https://dialnet. unirioja.es/descarga/libro/655308.pdf

Pérez-Escoda, N., Berlanga, V., y Alegre, A. (2019). Desarrollo de competencias socioemocionales en educación superior: evaluación del posgrado en educación emocional. Bordón 71 (1), 97-113. doi: https://doi.org/10.13042/Bordon.2019.64128

Rodríguez, J. F. (2016). La primera colonia escolar de vacaciones para niños pobres de las escuelas públicas madrileñas (1887). El futuro del pasado, 7, 407-439. doi: https:// doi.org/10.14516/fdp.2016.007.001.014

Rosenthal, J.A. (1996). Qualitative descriptors of strength of association and effect size. Journal of Social Service Research, 21(4): 37-59. doi: https://doi.org/10.1300/ J079v21n04_02

Saarni, C. (2000). Emotional Competence. A Developmental Perspective. En R. Bar-On y J. D. A. Parker (Eds.), The Handbook of Emotional Intelligence. Theory, Development, Assessment, and Application at Home, School, and in the Workplace. San Francisco, Ca: Jossey-Bass, 68-91.

Schön, D. (1998). El profesional reflexivo. Cómo piensan los profesionales cuando actúan. Barcelona: Paidós

Silva, M. A., Franco, M. G., Nobrega, N., y Porto, E. (2014) Outdoor Training como metodología para potenciar la inteligencia emocional en los niños y adolescentes. International Journal of Developmental and Educational Psychology INFAD Revista de Psicologia, 1 (5), 187-194. doi: http://dx.doi.org/10.17060/ijodaep.2014.n1.v2.458

Torrijos, P., Hernández, J. P., y Rodríguez, M. J. (2016). Desarrollo de competencias emocionales en los futuros docentes de Educación Secundaria: resultados de la aplicación de un programa formativo. Revista Educativa Hekademos, 2 (1), 35-43.

Trilla, J.( 1997). La Educación Fuera de la Escuela. Madrid: Ariel.

Fecha de recepción: 5 de diciembre de 2019.

Fecha de revisión: 22 de enero de 2020.

Fecha de aceptación: 10 de marzo de 2020. 
